Research Article

\title{
Research on Artificial Intelligence Interaction in Computer-Aided Arts and Crafts
}

\author{
Juqing Deng $(\mathbb{D}$ and Xiaofen Chen \\ School of Communication \& Art Design, Wuxi Institute of Arts and Technology, Yixing 214206, China \\ Correspondence should be addressed to Juqing Deng; dengjuqing@wxgyxy.edu.cn
}

Received 29 January 2021; Revised 19 March 2021; Accepted 17 May 2021; Published 27 May 2021

Academic Editor: Hsu-Yang Kung

Copyright (C) 2021 Juqing Deng and Xiaofen Chen. This is an open access article distributed under the Creative Commons Attribution License, which permits unrestricted use, distribution, and reproduction in any medium, provided the original work is properly cited.

\begin{abstract}
Background. With the continuous maturity of computer software and hardware technology, the theory and method of computeraided art design have developed rapidly. Objective. Applying artificial intelligence theory to computer-aided process art design is one of the newly developed research hotspots, and it is also the development trend of industrial design modernization. Methods. On the one hand, it can transplant the research results in the field of artificial intelligence into computer-aided art design, and on the other hand, it expands the application field of artificial intelligence, so that the two can be perfectly combined to promote common development. Results. With the development of artificial intelligence technology, computer art has gradually become a very active field, and a large number of computer art works are available every year. Conclusions. This paper briefly describes the basic concepts of computer-aided art design and artificial intelligence and discusses the application of artificial intelligence in computer-aided art design.
\end{abstract}

\section{Introduction}

Computer-aided arts and crafts art design are a new type of technology that applies computer technology to art and art design, freeing staff from tedious and complicated work. Let the traditional arts and crafts art design improve the quality of arts and crafts art products while improving work efficiency. With the continuous advancement of computer technology, the emergence of artificial intelligence has added new vitality to computer-aided arts and crafts, and the level and efficiency of art and art design have been improved as never before [1].

A supply chain is a network of retailers, distributors, transporters, storage facilities, and suppliers that participate in the production, delivery, and sale of a product to the consumer. It is typically made up of multiple companies who coordinate activities to set themselves apart from the competition. There are three key parts to a supply chain: supply focuses on the raw materials supplied to manufacturing, including how, when, and from what location. Manufacturing focuses on converting these raw materials into finished products. Distribution focuses on ensuring these products reach the consumers through an organized network of distributors, warehouses, and retailers.

In the application of electronic computers, hard devices and software related to image processing are increasing at a rate of $40 \%$ per year, that is, doubling every two years. The use of interactive computer-aided design system as an auxiliary tool for art creators to design arts and crafts has a very significant effect on reducing duplication of work, speeding up the design progress, enlightening creative ideas, and enriching the content of the work. With the development of artificial intelligence technology, computer art has gradually become a very active field, and a large number of computer art works are available every year $[2,3]$. Many arts and crafts have the characteristics of repeating on a flat surface, which has often appeared in ancient art treasures. Using some simple graphic basic units, combined with the general principle of composition and the automatic generation technology of interlocking graphics, you can produce certain practical arts and crafts on computer colour monitors or ordinary colour (or black and white) TV screens 
(graphic, for silk stretch, flower cloth, straw, batik, carpet, and other design use). The use of the joint money graphic automatic generation technology can automatically interlock and copy the mosaic graphics without any drying and overlapping and can be used for metal cutting, wood, leather, etc. without cutting, stamping, and cutting and processing and can also be used for the design of floor tiles, art floors, wallpapers, etc. [3].

Mobile information system is based on mobile equipment, through the high-performance wired and wireless communications networks for information and data transmission, to provide distributed GIS of the locationbased information services anytime and anywhere. It integrates GIS, GPS, mobile communication and computer networks, and so on, and the advantages of these technologies will be integrated to information transfer through the high-performance communication networks, so that we can achieve mobile information of the industry and public information service. It also provides a convenient and economical way of technology for users on the exchange, access, and share and publish of information based on location, forming a GIS application way for portable terminals such as mobile phones. Mobile information system is especially applicable to commercial trade, government, tele-com, municipal construction, and other industries whose data have highly mobility and changes frequently.

Applying artificial intelligence theory to computer-aided process art design is one of the newly developed research hotspots, and it is also the development trend of industrial design modernization. On the one hand, it can transplant the research results in the field of artificial intelligence into computer-aided art design, and on the other hand, it expands the application field of artificial intelligence, so that the two can be perfectly combined to promote common development. With the development of artificial intelligence technology, computer art has gradually become a very active field, and a large number of computer art works are available every year. This paper briefly describes the basic concepts of computer-aided art design and artificial intelligence and discusses the application of artificial intelligence in computer-aided art design.

The specific contributions of this paper include the following:

(1) Transplant research results in the field of artificial intelligence into computer-aided art design

(2) Introduce the basic concepts of computer-aided art design and artificial intelligence

(3) Discuss the application of artificial intelligence in computer-aided art design

The rest of this paper is organized as follows. Section 2 discusses artificial intelligence, followed by application of artificial intelligence in the design of computer-aided arts and crafts in Section 3. Theoretical model of artificial intelligence in the design of computer-aided arts and crafts is discussed in Section 4. Section 5 shows result analysis and discussion, and Section 6 concludes the paper with summary and future research directions.

\section{Artificial Intelligence}

Artificial intelligence, referred to as AI, is a new technical science that studies and develops theories, methods, techniques, and applications that simulate, extend, and expand human intelligence. The areas of the artificial intelligence are shown in Figure 1 [4]. The use of artificial intelligence can make various computer devices more humanized, extend and expand human intelligence, and realize the highest-level application of computers. The application of computer technology in people's lives has been expanding, and people have put forward new requirements for the ability of computers. The emergence of artificial intelligence technology has brought computer technology to a new level.

At present, there are mainly three research directions of artificial intelligence, namely intelligent interface, data mining, subject and multiagent system. Intelligent interface is mainly to study how people can communicate with computers more conveniently and intuitively; data mining mainly studies how to dig out people from complex, diverse, incomplete, large, fuzzy, and random application data. The process of knowing is having important or potentially meaningful information; the subject refers to the subject of artificial intelligence, which has certain independence, and mainly studies the ability of artificial intelligence to face the entity in the selection layer of belief, desire, intention, and ability. At present, the research of artificial intelligence has made some achievements in the reasoning ability and is developing towards the fuzzy processing function and the parallel processing function. The future development direction of artificial intelligence is artificial neural network. The advancement of artificial neural network will greatly promote the progress of society [5].

\section{Application of Artificial Intelligence in the Design of Computer-Aided Arts and Crafts}

The concept of computer-aided arts and crafts was put forward in the 1970s, and it has been widely concerned in the development process. It has experienced multiple stages of interaction, derivation, and generative, comprehensive, and intelligent progress [3]. For the time being, intelligence is the focus of computer-aided process development and the direction of future development. The biggest role of computeraided arts and crafts in work is to replace some manual and repetitive operations, freeing up a large number of manual labor, so that people can put more energy into the research and development of new technologies and new arts and crafts. Under the help of computer-aided arts and crafts, people can realize the optimal allocation of limited resources, while reducing production costs and improving production efficiency. Computer-aided arts and crafts not only provide important support in production, but also solve a lot of designer's troubles in design. Some young designers who lack design experience can use computer-aided arts and crafts technology to improve the design level, improve the design grade, and alleviate the large demand for designers with rich design experience. 
3.1. Intelligence. The most outstanding advantage that artificial intelligence brings to computer-aided arts and crafts design is intelligence. Computer-aided arts and crafts technology still has some links in the arts and crafts. It is difficult to get rid of the scope of auxiliary tools, but the addition of artificial intelligence has changed this. The current status makes computer-aided process design have certain intelligent analysis capabilities. Artificial intelligence is still in the state of research, and the application of artificial intelligence in computer-aided process design is mainly manifested in intelligent reasoning. In practical applications, the application forms of artificial intelligence are not unique, but various applications penetrate each other to form a comprehensive application form. In the field of computeraided design, computer-aided mechanical product design is the most studied, while computer-aided art design and mechanical product design have certain convergence, so it can be inferred that the architecture of intelligent computeraided art design includes science (layer, information technology layer, and intelligent design layer).

Applying artificial intelligence technology to the development of computer-aided art design (CAPP) system, CAPP system simulates people's thinking mode in knowledge acquisition, knowledge reasoning, etc., solves complex art and art design problems, and makes it "human intelligence". The feature is intelligent CAPP, which is an application of AI in CAPP. In the development of intelligent CAPP system, there are also intelligent methods such as fuzzy reasoning and chaos theory [6]. In practical applications, a variety of intelligent technologies are often combined to exert their own specialties, such as the characteristics of artificial neural networks with perceptual image thinking. Methods of fuzzy inference and other characteristics of logical thinking interpenetrate and combine, can play a complementary role, and improve the level of intelligence. There are few researches on CAPP design theory, and there are many researches on mechanical product design theory. The design theory and method consists of three parts: design theory foundation layer, design tool, and support technology platform layer. CAPP design theory and mechanical product design theory have commonality and particularity, especially in the intelligent design method. Therefore, it is considered that the intelligent CAPP design theory and method architecture consists of three layers, namely the foundation science layer, information technology layer, and intelligent design method layer.

3.2. Artificial Neural Network Technology. Art and craft design is a relatively large and cumbersome category. There are many uncertain factors in the design and production process. These uncertain factors are likely to cause problems in computer-aided art design and affect the efficiency of the entire design and production. The emergence of artificial neural network technology has improved the skills of computer-aided arts and crafts (CAPP) design, and these problems have been effectively controlled. In the process of dealing with the problem of the network world, the artificial neural network simulates the principle of the biological nervous system. It is composed of a large number of nonlinear processing units. The units are highly paralleled by modern information technology. The artificial neural network has several characteristics when dealing with problems: fabric storage, parallel processing, self-organization and selflearning, and associative memory. The biggest advantage of applying artificial neural network technology to computeraided art design is that this technology can adapt and adjust the system. When checking the initial basic values and the actual conditions inside the system, the neural network will monitor its own adaptability. The specific method of monitoring is to deduct and adjust from the beginning of the neural network to each node in the back and then push and adjust from the end of the neural network to better adapt to the network application.

In the design of product art and craft, there are many uncertain factors, which need to be solved by experience. The early establishment of CAPP system based on purely relying on group technology cannot solve the problem of obtaining this discrete knowledge well, design a search or derivative system. In recent years, the application of artificial intelligence technology in the development of CAPP system has made CAPP technology a great development [7]. Artificial neural network technology is one of the major applications of AI in CAPP system. Artificial neural network is an objective object that deals with the real world according to the principle of biological nervous system. It is composed of a large number of simple nonlinear processing units connected in parallel, with distributed storage of information, parallel processing, self-organization and self-learning, associative memory, etc. Characteristics are multilayer feed forward network error back propagation algorithm [8], as shown in Figure 2. The back-propagation algorithm is an algorithm for supervising the training of multilayer neural networks. Each training paradigm is calculated by two passes in the network: the first pass is calculated forward, from the input layer, and the layers are transmitted and processed and then generated. This picture shows the execution process of the neural network completely. Giving a picture, through the network supervision operation, the gradient is obtained and the gradient is updated, and finally the accurate result is obtained (an output, and an error vector of the difference between the actual output and the desired output; the second pass backwards, from the output layer to the input layer, using the error vector to modify the weight layer by layer).

3.3. Rough Set Technology. The rough set technology is an application form of artificial intelligence fuzzy control technology, which can effectively solve the ambiguity problem in computer-aided art design and achieve the purpose of solving the problem through comparative analysis. Artificial intelligence first lists the ambiguity problem as a table in the form of parameters, arranges the influencing factors corresponding to these problems according to the degree of influence, then horizontally lists some possible problems to set the weights, and finally eliminates them by certain algorithms. The redundant 
parameters in the problem can finally find the correct answer to the fuzzy problem [9].

Rough set technology is another application of artificial intelligence in computer-aided art design. Rough set theory is a mathematical tool that is good at dealing with ambiguous and uncertain problems. In theory, "knowledge" is considered as a kind of classification ability for objects. Usually, a two-dimensional decision table is used to describe the domain information, and the column represents an attribute, a line represents an object, and each line represents a piece of information about the object. Attributes are divided into conditional attributes and decision attributes. Objects in the domain are divided into decision classes with different decision attributes according to different condition attributes. In the CAPP system, the expert system can be constructed by rough set theory, and the knowledge is acquired and optimized. The basic idea is to express the processing characteristics of various parts and the known processing methods into the form of conditional attributes and decision attributes. A part, a plurality of parts, forms a two-dimensional table, quantifies the attributes, organizes the decision table, and then uses a certain reduction algorithm to reduce the attribute set and attribute values, removing the redundant condition attributes and decision rules, and obtaining minimization of the set of decision rules, and when you input the machining features of the parts to be processed, you can get optimized processing techniques.

3.4. Genetic Algorithm. The genetic algorithm is influenced by Darwin's theory of evolution. In the computer field, the scientific principle of genetics is used to simulate the process of biological evolution, and the screening of arts and crafts is completed. The genetic algorithm classifies each target art. All possible target arts and crafts are called domain, and the art and craft solutions are divided into different populations according to different types. The artisan solution contained in the domain will give a unique gene coding. In the case of genetic algorithm, each process art of different populations is compared and analyzed in the way of survival of the fittest, and the first generation of populations is crossed and evolved by genetic operators to produce the next generation of populations, and so on, to the last generation of the population which is the optimal solution.

Genetic algorithm is another application of artificial intelligence in computer-aided art design system [10]. Genetic algorithm is a computational model that simulates the evolutionary process of Darwin's genetic selection and natural elimination. It is a method to search for optimal solutions by simulating natural evolutionary processes. The genetic algorithm begins with a population that represents a potential set of problems, while a population consists of a number of individuals genetically encoded, each of which is actually an entity with chromosomal features. Therefore, at the outset, it is necessary to implement mapping from phenotype to genotype, that is, encoding work, such as binary encoding. After the birth of the first generation, according to the principle of survival of the fittest and the principle of survival of the fittest, evolutionary generation produces better and better approximate solutions. In each generation, individuals are selected according to the fitness of individuals in the problem domain and with the help of natural genetics. Genetic operators perform combination of crossovers and mutations to produce populations that represent new solution sets. This process will lead to a population of natural evolution like the descendant population that is more adapted to the environment than the previous generation, and the best individual in the last generation population is decoded, which can be used as a problem approximate optimal solution.

In the last three years, many methods are proposed to handle the computer aids arts and crafts; here we introduced three outstanding methods such as GNNA [11], RNNA [12], and CNNA [13], which can be used to solve the related works taking different kinds of network structures. GNNA is a graph-based network that build connections between different risk nodes that utilize the existing crafts pictures to design new arts and crafts. And RNNA uses a specific loss structure to keep the similarity of real and predicted crafts design that can make sure that the computer designed crafts are as similar as the real ones. CNNA is the basic model that needs more computation consuming to obtain the desiring performances, which can only obtain the crafts as simple as possible. However, these methods have their disadvantages respectively. GNNA is too slow, RNNA is so complicated, and CNNA also needs more spaces. They have their own corresponding advantages and shortcomings, and our proposal can handle these problems as much as possible, so we compared them with our proposal.

In this paper we utilize the entropy loss function to build the model for our research problems. It can be defined as follows:

$$
\operatorname{loss}(x, y)=\sum_{i=1}^{n}-p_{i} \log \left(1-p_{i}\right)
$$

where $x$ and $y$ are represented as the real arts and crafts' score and difficulty and $y$ means the predicted score and difficulty of our proposal. $P_{i}$ means the probability of them when they are similar. The bigger the value of the loss is, the worse our proposal performed. And our proposal is used to train a model that fits the real and predicted arts and crafts, so that the machine can assist the arts and crafts designed.

And compared with the three methods, our proposal can deal with the problems easily and we also need a smaller computation space to build our model. However, our model may obtain a relative lower accuracy than others sometimes which may lead the prediction to be unstable.

\section{Theoretical Model of Artificial Intelligence in the Design of Computer-Aided Arts and Crafts}

4.1. Graphical Representation and Data Structure. The goal of the system is to generate a complete picture on the display called "picture". The picture is superimposed by one or several basic patterns (BG) copied by interlocking transformation (I): 


$$
\text { PICTURE }=F\left(I_{1}(B G), I_{2}(B G), I_{3}(B G), \ldots\right) .
$$

$F$ represents the superposition relationship and $I_{i}$ is one of the nine basic interlocking transformations, $I_{i} \in\left\{I_{1}, I_{2}, I_{3}, I_{4}, \ldots\right\}$.

Since the postgenerated graphics may partially cover the original graphics when the overlay is performed, the overlay is ordered. The basic graphics (BG) is a superposition of a series of line segments $\left(L_{i}\right)$. In the graphics library, the storage structure is

$$
B G=\left(N \$, X, U, V, N, H, Z_{0}-Z_{5}, L_{0}, L_{1}, L_{2}, \ldots, L_{N}\right) .
$$

$N \$$ is the name of the basic graphic composed of strings, which is necessary for the graphics inventory to retrieve and print graphic data, $x \in\{0,1\}$.

When $X=0$, the pattern is high-resolution, with only two levels of brightness and darkness. When $X=1$, the pattern is low-resolution, with 15 colors or 16 gray levels. $N+1$ is equal to the number of line groups the basic figure has. $\mathrm{H}, \mathrm{U}$, and $\mathrm{V}$ are graphical interlocking parameters. $Z_{0}-Z_{5}$ are optional parameters of the basic graphics, which may represent the number of times the graphics are copied in the horizontal and vertical directions or may represent the background or background brightness of the graphics.

Each line group $\left(L_{i}\right)$ is sequentially connected by a series of ordered vertices $\left(V_{i}\right)$ using straight line segments generated by the complement method. When $X=l$ (colour or multigradation), the line group should have a colour or brightness attribute $\left(B_{i b}\right)$, and when the number of vertices of the line group $L_{i}$ is large or equal to four and closed (i.e., the first and last vertices coincide), the line group constitutes the boundary of one (or more) enclosed area and thus also has the colour or brightness properties of the inner area $\left(B_{\mathrm{ir}}\right)$.

$$
L_{i} \in\left[N_{i} \times B_{i b} \times\left\{V_{0}\right\} \times\left\{V_{1}\right\} \times\left\{V_{2}\right\} \times \cdots \times\left\{V_{N i}\right\} \times B_{\text {ir }}\right] .
$$

$x$ is the Cartesian product operator of the set, and items in square brackets may or may not appear.

$$
N_{i}=\{-1,0,1,2, \ldots\} \text {. }
$$

$N_{i}+1$ is the number of vertices in the line group. When $N=-1$, it means an empty line group whose number of vertices is 0 , and $L_{i}$ has no other parameters.

$$
\begin{aligned}
B_{i b} & =\{1,2,3,4, \ldots, 16\}, \\
B_{\text {ir }} & =\{0,1,2,3, \ldots, 16\} .
\end{aligned}
$$

When $B_{\text {ir }}=0$, the inside of the enclosed area is not filled.

The vertex $V$ is determined by its pair of coordinate values in a Cartesian coordinate system:

$$
V_{i}=\left(X_{i}, Y_{i}\right) \text {, }
$$

where $X_{i}$ and $Y_{i}$ are integers.

\subsection{Graphics Transformation and Automatic Generation} Technology of Interlocking Graphics. The process of spreading the entire plane with congruent graphics without gaps and overlapping portions is called tessellation, and the inlaid graphics are called interlocking graphics. The vertices of the polygons (the number of sides must be greater than two, less than seven) can be embedded into the interlocking graph. The symmetry principle and the congruent linear transformation of the graph can form the interlocking transformation mode of the nine graphics. Nine ways can be derived from 29 interlocking graphics. The basic principle of graphical linear transformation and the algorithm for generating interlocking graphics are briefly introduced below [14].

For linear transformation,

$$
\left\{\begin{array}{l}
x^{\prime}=a x+b y+c, \\
y^{\prime}=d x+e y+e .
\end{array}\right.
$$

It can be expressed as a standard method of $3 \times 3$ matrix :

$$
\left[x^{\prime}, y^{\prime}, 1\right]=[x, y, 1] \cdot\left[\begin{array}{lll}
a & d & 0 \\
b & e & 0 \\
0 & f & 1
\end{array}\right] .
$$

The most basic three linear transformations are as follows.

(1) Translation transformation:

$$
\left[x^{\prime}, y^{\prime}, 1\right]=[x, y, 1] \cdot\left[\begin{array}{ccc}
1 & 0 & 0 \\
0 & 1 & 0 \\
x_{k}^{\prime}-x_{k} & y_{k}^{\prime}-y_{k} & 1
\end{array}\right] .
$$

$\left(x_{k}, y_{k}\right)$ and $\left(x_{k}^{\prime}, y_{k}^{\prime}\right)$ indicate the coordinates of any known point in the before and after translation, respectively. The translation transformed by (10) is transformed into a $\mathrm{T}$ transform.

(2) Proportional transformation:

$$
\left[x^{\prime}, y^{\prime}, 1\right]=[x, y, 1] \cdot\left[\begin{array}{ccc}
P_{x} & 0 & 0 \\
0 & P_{y} & 0 \\
0 & 0 & 1
\end{array}\right] \text {. }
$$

(3) Rotation transformation centered on the origin of the coordinates:

$$
\left[x^{\prime}, y^{\prime}, 1\right]=[x, y, 1] \cdot\left[\begin{array}{ccc}
\cos \theta & -\sin \theta & 0 \\
\sin \theta & \cos \theta & 0 \\
0 & 0 & 1
\end{array}\right] \text {. }
$$

When $\theta>0$, it means clockwise rotation. The linear transformation used in the system can be successively performed by the above three basic transformations, and the transformed cascade matrix can be represented by the product of the basic transformation matrix. The linear transformations used in this system are as follows. 
(4) A rotation transformation cantered on $\left(x_{c}, y_{c}\right)$, which is made up of $\left(-x_{c},-y_{c}\right)$ translation, $\theta$ rotation $(\theta>0$ is clockwise), and $\left(x_{c}, y_{c}\right)$ translation cascade:

$$
\left[x^{\prime}, y^{\prime}, 1\right]=[x, y, 1] \cdot\left[\begin{array}{ccr}
\cos \theta & -\sin \theta & 0 \\
\sin \theta & \cos \theta & 0 \\
-x_{c} \cos \theta-y_{c} \sin \theta+x_{c} & x_{c} \sin \theta-y_{c} \cos \theta+y_{c} & 1
\end{array}\right]
$$

When $\theta$ is equal to $180^{\circ}, \pm 120^{\circ}, \pm 90^{\circ}$, and $\pm 60^{\circ}$, the transformation determined by (13) is called $\mathrm{C}, \mathrm{C}_{3}$, $\mathrm{C}_{4}$, and $\mathrm{C}_{5}$ transformation. Table 1 shows the typical combination of $\mathrm{C}$ series and $\mathrm{T}$, and their variations.

(5) A reflection transformation with the line $y=x$ as the axis, which is $45^{\circ}$ rotation, $(1,-1)$ ratio and $-45^{\circ}$ rotation three basic transformation cascades.

(6) Make reflective translation transformation, after mirror reflection of the horizontal or vertical axis of the point $\left(x_{\mathrm{c}}, y_{\mathrm{c}}\right)$, then translate the point to point $\left(-\mathrm{x}_{\mathrm{c}},-\mathrm{y}_{\mathrm{c}}\right)$, which is made up of $\left(-x_{k},-y_{k}\right)$ translation, $\left(P_{x}, P_{\mathrm{y}}\right)$ scale and $\left(x_{\mathrm{k}}, y_{\mathrm{k}}\right)$ translation cascade. For horizontal axis reflection, $P_{x}=1, P_{y}=-1$, and for vertical axis reflection, $P_{x}=-1, P_{y}=1$. At this time, the reflection translation transformation can be expressed as

$$
\begin{aligned}
& {\left[x^{\prime}, y^{\prime}, 1\right]=[x, y, 1] \cdot\left[\begin{array}{ccc}
1 & 0 & 0 \\
0 & -1 & 0 \\
x_{k}^{\prime}-x_{k} & y_{k}^{\prime}+y_{k} & 1
\end{array}\right],} \\
& {\left[x^{\prime}, y^{\prime}, 1\right]=[x, y, 1] \cdot\left[\begin{array}{ccc}
-1 & 0 & 0 \\
0 & 1 & 0 \\
x_{k}^{\prime}+x_{k} & y_{k}^{\prime}-y_{k} & 1
\end{array}\right] .}
\end{aligned}
$$

The transforms determined by (14) and (15) are referred to as $G_{\mathrm{h}}$ transform and $G_{\mathrm{v}}$ transform, respectively. Among the seven transformations T, C, $\mathrm{C}_{3}, \mathrm{C}_{4}, \mathrm{C}_{6}, G_{\mathrm{h}}$, and $G_{\mathrm{v}}$ used in the construction of the interlocking pattern, except for the $\mathrm{C}$ transformation, the other transformations appear in pairs. Only half of the line group formed by the $\mathrm{C}$ transform is independent, so it forms a curve pair itself. In the TT, $\mathrm{C}_{3} \mathrm{C}_{3}$, $\mathrm{C}_{4} \mathrm{C}_{4}, \mathrm{C}_{6} \mathrm{C}_{6}, \mathrm{G}_{\mathrm{h}} \mathrm{G}_{\mathrm{h}}$, and $\mathrm{G}_{\mathrm{v}} \mathrm{G}_{\mathrm{v}}$ pair, only one curve is independent and can be determined by the user. The other subordinate line is separated by the above all. It is obtained by linear transformation, so that in each basic unit of the interlocking graphics, only half of the edges are independent, and the other half of the edges are generated by the independent edges by the congruent linear transformation. The $\mathrm{T}$ transform and the $\mathrm{G}$ transform cannot be combined with the $\mathrm{C}_{3}, \mathrm{C}_{4}, \mathrm{C}_{6}$ transforms.

For the case of converting each basic graphic unit into an interlocking mosaic pattern, it is necessary to find the group that can be displayed on the translated and complex quadrilateral set, and determine a parallelogram grid, which consists of two displacement vectors, the level in one level is determined, the length is $\mathrm{H}$, and the other has a horizontal component and a vertical component. Volume V.H, U and $\mathrm{V}$ are the interlocking parameters mentioned above. When the line cluster moves enough times along the corresponding parallelogram grid, several congruent interlocking graphs are copied on the entire display screen.

$$
\begin{aligned}
& H(\theta, x, y \mid u, v)=h(\theta \mid u) \prod_{n=1}^{N} h\left(x_{n} \mid u\right) p\left(y_{n} \mid x_{n}, v\right) \\
& H(y \mid u, v)=\int h(\theta \mid u)\left(\prod_{n=1}^{N} \sum_{z_{n}} h\left(x_{n} \mid \theta\right) h\left(y_{n} \mid x_{n}, v\right)\right) \mathrm{d} \theta .
\end{aligned}
$$

Table 2 shows the typical combination of $\mathrm{C}$ series, $\mathrm{T}, \mathrm{G}$, and their variations.

\subsection{Structure and Main Functions of the Graphic Design} System. The equipment used in this system is CROMEMCO52 microcomputer system, including DAZZLER graphic output interface board and an external colour monitor (can also use ordinary TV sets). To improve the interaction between graphic designers and systems, fully to play the function of the original graphics software resources of the system, the extended BASIC language (including several basic graphic command statements) and the corresponding GRAPHX $\bullet$ COM command file software interface are selected. It takes about one to two minutes to output an interlocking graphic picture composed of four to sixteen basic units on the monitor using the graphic design software package, and each basic unit can have up to 21 line groups [15]. In the case of low resolution, these line groups can have 15 different colors, and each line group can be connected by up to 21 vertices. In view of the fact that the system update memory capacity in the main memory is $\mathrm{ZK}$ byte RAM, the resolution is low, so it is not practical to increase the number of lines and vertices of the basic unit of the graphic, and one picture can be interlocked by multiple frames [16]. The graphics are superimposed, each interlocking graphic is interlocked by multiple graphic basic units, and the system has a large area automatic colour filling function, so the number of vertices and the number of line groups can satisfy the minimum actual needs. For more advanced graphics output devices, the system can be used with a little modification.

Since the DAZZLER graphical interface allows the program to easily and flexibly select two different 


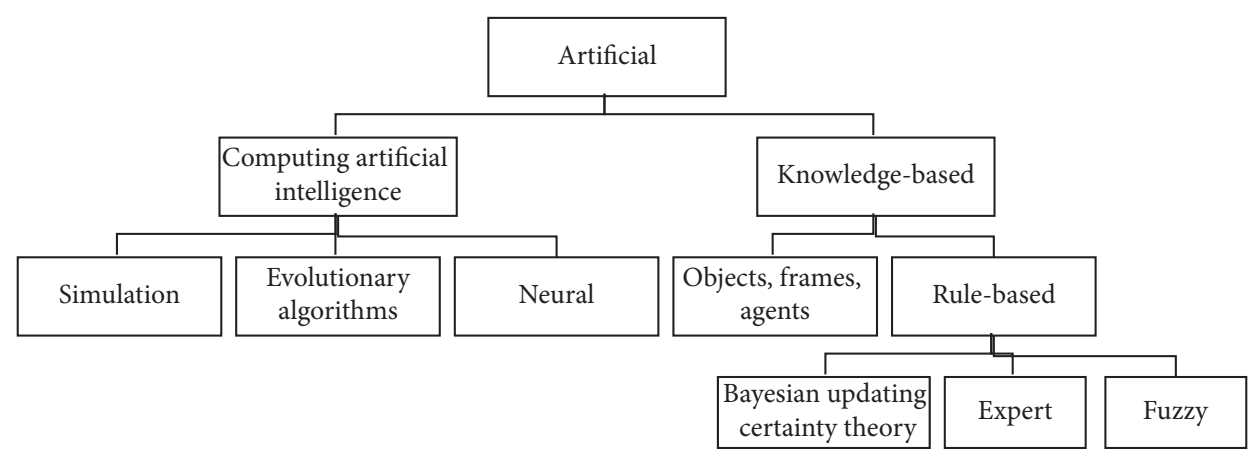

FIgURE 1: The areas of artificial intelligence.

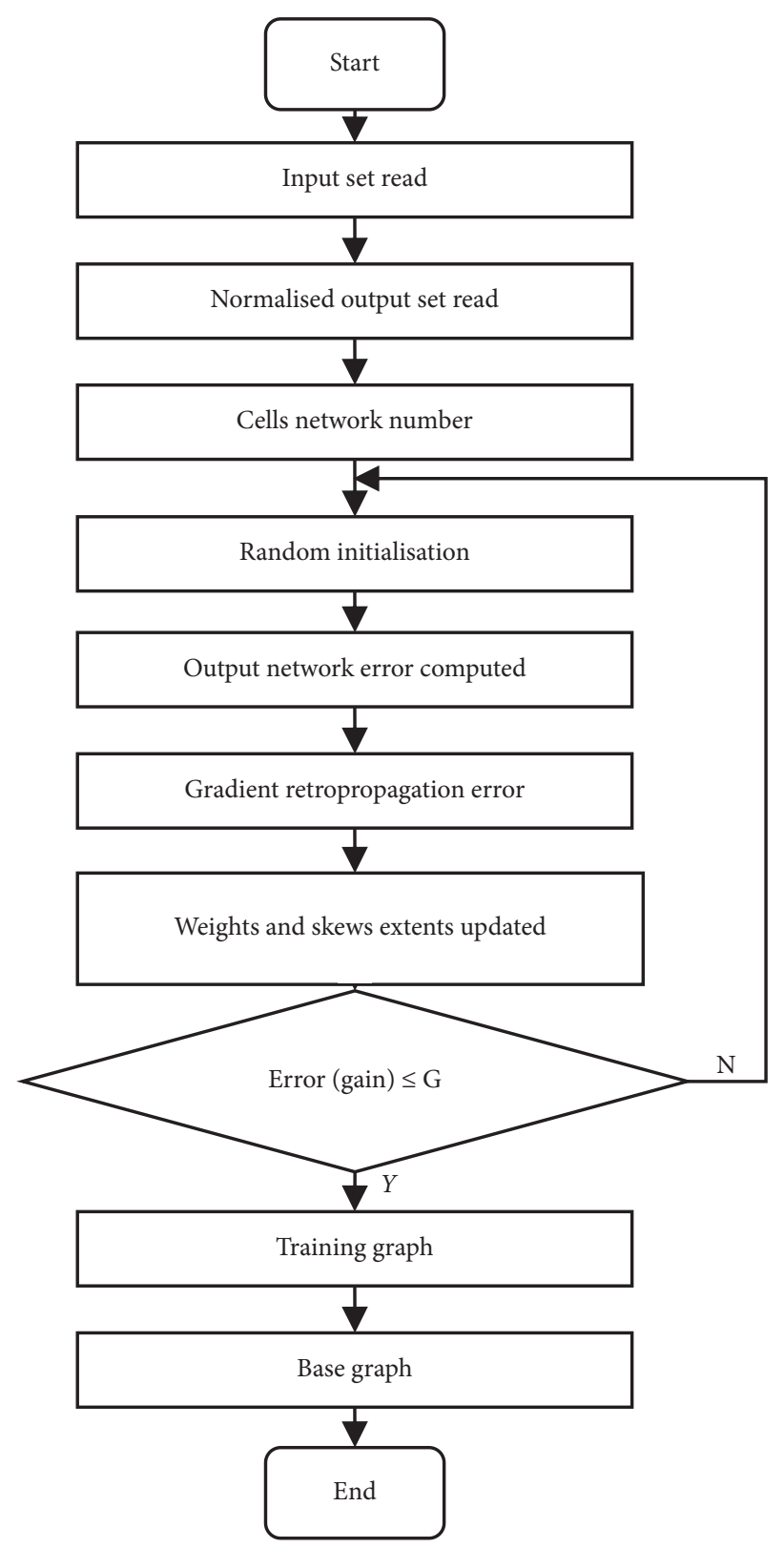

FIgURE 2: The backpropagation algorithm. 
resolutions, the graphic designer can design two different types of graphics as needed.

4.3.1. Colour or Multigradation (Low Resolution). When the graphic has colour or multigradation (low resolution), the internal lines of the basic unit of the graphic are mainly used, and various arts and crafts graphics are designed by interlocking and copying the interior of the closed area. Since the interlocking graphics have the reciprocity of periodic repetition, it is easy to ensure that two consecutive or quadrilateral continuous images are obtained. This is very convenient for the design of many arts and crafts [17]. Since the pattern designer can arbitrarily choose the interlocking mode and the interlocking translation parameters, it is convenient to modify the ratio of the graphics in the $x$ direction and the $y$ direction and the number of copies of the basic unit and flexibly shift the entire screen up and down or left and right during copying [18]. Reflecting with the $y=x$ line as the axis, you can also flexibly modify the colour or brightness of any line group or all line groups, specify the background colour of the screen or modify the colour inside a certain closed area, and classify according to certain graphics. The sequence is superimposed to form a new picture, so even if you input a very simple graphic basic unit, you can change thousands of different patterns to make it easier for designers to compare the effects of various structures and tones. The system also has a loopshaped database automatic design function, which can provide graphic designers with some optional art graphics to inspire the creative ideas of the art designers. Therefore, these graphics are suitable for silk, flower cloth, parquet floor, carpet, and wallpaper (design of arts and crafts such as straw and straw) $[19,20]$.

4.3.2. Black and White (High Resolution). When the graphic is designated as black and white (high resolution), the interlocking graphic is the main working mode by the boundary line of the graphic and the internal line. Since such graphics often form a gap-free, nonoverlapping interlocking pattern by the basic graphic unit translation, the system is applied in the processing of sheet metal processing (such as cutting and punching of various metal sheets, wood, leather, and paper) [21]. This can help designers save raw materials and reduce product costs. For most graphics, even if there is no gap at all, the closest basic unit can be found in 29 interlocking modes, so that the machining allowance is reduced as much as possible $[22,23]$.

In the nine main interlocking transformation modes, several different shapes of graphics can be skillfully combined to form a basic unit of interlocking graphics. In turn, the basic unit of the interlocking graphics can also flexibly use internal lines as needed. Divided into several different shapes of graphics. This kind of interlocking graphic design method based on boundary line has certain uses in building block design and modular upper floor planning and packaging design of irregular products [24]. It can also be used for building materials such as floor tiles and wallpaper (designed for use in batik, lace, and other crafts). Because only half of the boundaries of this type of graphics are independent, the designer only needs to input half of the basic graphics plus the internal lines, so the system can copy multiples at any scale (only limited by display size and resolution). Such resulting graphics, thus greatly improving the design efficiency, and because the other half of the boundary line of the basic unit is generated by different interlocking methods, often have the effect that the designer did not expect [25]. Any graphic designer with elementary mathematics and a little English can use this graphic design program system to design various graphics after several hours of learning. Nine kinds of interlocking transformation graph generation programs exist in the floppy disk memory respectively. The graphic designer uses the host console keyboard and the character display terminal to perform man-machine dialogue. After selecting the interlock mode, three different methods can be used for graphic design [26].

(1) The Interaction Method Using Keyboard Input. After the designer specifies the resolution and background colour of the graphic (for colour or multigravy graphics) through the keyboard, the system draws the background colour on the colour monitor and displays the coordinate origin and the rough scale of the $x$ and $y$ axes [27]. You can enter the coordinates of known vertices and independent line group keys. For colour graphics, you also specify the colour of each line group. For the closed area, you also specify whether to fill and fill the code. Each time a key is entered, the system immediately displays it on the monitor and automatically joins the line group. After the independent line group is finished, the system immediately copies the corresponding subordinate line group to form a complete boundary line. The designer can then enter the internal line set. After the input process is finished, the system automatically copies and copies any number of graphic basic units in the $x$ and $y$ directions according to the designer's request to form an interlocking graphic picture [28]. During the entire input process, if the designer wants to change a certain line group that has just been input, the keyboard group can be easily erased from the screen by the keyboard, or the line group can be temporarily reserved for modification as a comparison, and when the interlock is copied, the invalid line group does not appear. If the designer believes that the graphics copied by the chain is necessary, all the graphic data can be printed for future copying or analysis. The system can automatically organize all the data and related parameters into data files according to the specified format and data structure. Into the disk, recopy on the monitor when needed $[29,30]$.

(2) Graphics Library Method. All data and partial interlocking parameters of the graphic basic unit are predetermined by the designer and organized into data files according to the specified data structure and stored in the disk to form a graphic library, which is called out from the disk during design and is spliced or superimposed on the monitor. Synthesize new pictures [31].

(3) Intelligent Graphic Database Automatic Design Method. According to certain composition rules and their own preferences, the art graphic designer combines the basic unit 
TABle 1: The typical combination of $\mathrm{C}$ series and $\mathrm{T}$.

\begin{tabular}{|c|c|c|c|c|c|c|c|}
\hline Transformations & $\begin{array}{c}\text { Known } \\
\text { points }\end{array}$ & $\begin{array}{l}\text { Lonely } \\
\text { points }\end{array}$ & Belonging points & $\begin{array}{c}\text { Lonely } \\
\text { line }\end{array}$ & $\begin{array}{c}\text { Belonging } \\
\text { line }\end{array}$ & Variation & Conditions \\
\hline TTTTT & $\begin{array}{l}x(0)=0 \\
y(0)=1 \\
x(3)=50 \\
y(3)=10\end{array}$ & $\begin{array}{l}x(1) \\
y(1) \\
x(4) \\
y(4)\end{array}$ & $\begin{array}{l}x(2)=x(1)+20 \\
y(2)=y(1)+3 \\
x(5)=x(2)-x(3)+2 \\
y(5)=Y(2)-y(3)\end{array}$ & $\begin{array}{l}01 \\
13 \\
23\end{array}$ & $\begin{array}{l}42 \\
54 \\
04\end{array}$ & TTTT & $\begin{array}{l}x(8)=y(8)=0 \\
x(3)=0.25 x(1) \\
y(3)=0.25 y(1)\end{array}$ \\
\hline TCСТCC & $\begin{array}{l}x(0)=0 \\
y(0)=0 \\
x(6)=25 \\
y(6)=0\end{array}$ & $\begin{array}{l}x(1) \\
y(1) \\
x(8) \\
y(8) \\
x(3) \\
Y(3)\end{array}$ & $\begin{array}{l}x(2)=0.125 x(1)+0.125 x(3) \\
y(2)=0.125 y(1)+0.125 y(3) \\
x(5)=0.25 x(1)+25 \\
y(5)=0.25 y(1) \\
x(4)=0.125 x(3)+0.125 x(1)+15 \\
y(4)=0.2 y(2)\end{array}$ & $\begin{array}{l}01 \\
12 \\
34 \\
67\end{array}$ & $\begin{array}{l}23 \\
65 \\
45 \\
78\end{array}$ & $\begin{array}{c}\text { TCTCC } \\
\text { TCTC } \\
\text { CCC }\end{array}$ & $\begin{array}{l}x(8)=y(8)=0 \\
x(3)=0.25 x(1) \\
y(3)=0.25 y(1)\end{array}$ \\
\hline $\mathrm{C}_{3} \mathrm{C}_{3} \mathrm{C}_{3} \mathrm{C}_{2} \mathrm{C}_{2}$ & $\begin{array}{l}x(0)=0 \\
y(0)=0 \\
x(2)=10 \\
y(2)=27 \\
x(4)=25 \\
y(4)=-27\end{array}$ & $\begin{array}{l}x(1) \\
y(1)\end{array}$ & $\begin{array}{l}x(3)=0.1 x(1)-0.322 x(3)+47 \\
y(3)=0.35 x(1)-0.18 y(3) \\
x(5)=-0.2 x(1)+0.23 y(1) \\
y(5)=-0.23 x(1)-0.1 y(1)\end{array}$ & $\begin{array}{l}12 \\
01 \\
34\end{array}$ & $\begin{array}{l}05 \\
54 \\
34\end{array}$ & $\mathrm{C}_{3} \mathrm{C}_{3} \mathrm{C}_{2}$ & $\begin{array}{l}x(2)=x(1) \\
y(2)=y(1)\end{array}$ \\
\hline
\end{tabular}

Table 2: The typical combination of $\mathrm{C}$ series, $\mathrm{T}$ and $\mathrm{G}$.

\begin{tabular}{|c|c|c|c|c|c|c|c|}
\hline Transformations & $\begin{array}{l}\text { Known } \\
\text { points }\end{array}$ & $\begin{array}{l}\text { Lonely } \\
\text { points }\end{array}$ & Belonging points & $\begin{array}{l}\text { Lonely } \\
\text { line }\end{array}$ & $\begin{array}{c}\text { Belonging } \\
\text { line }\end{array}$ & Variation & Conditions \\
\hline \multirow{4}{*}{ TGh1 Ch1TGh2Gh2 } & $x(0)=0$ & $x(1)$ & $x(3)=0.2 x(1)+20$ & 01 & 05 & \multirow{4}{*}{$\mathrm{G}_{\mathrm{h} 1} \mathrm{G}_{\mathrm{k} 1} \mathrm{G}_{\mathrm{h} 2} \mathrm{G}_{\mathrm{k} 2}$} & \multirow{4}{*}{$x(1)=y(1)=0$} \\
\hline & $y(0)=0$ & $y(1)$ & $y(3)=0.3 y(1)$ & 23 & 43 & & \\
\hline & $x(6)=35$ & $y(2)$ & $x(5)=0.2 x(2)-0.2 x(1)$ & 12 & 34 & & \\
\hline & $\begin{array}{l}y(6)=0 \\
y(7)=8.4\end{array}$ & $y(5)$ & $y(5)=0.2 y(1)-0.1 y(2)$ & & & & \\
\hline \multirow{5}{*}{ TCCTGhGh } & $x(0)=0$ & $x(1)$ & $x(2)=0.1 x(1)-0.2 x(3)$ & 12 & 32 & \multirow{5}{*}{$\mathrm{TCTG}_{\mathrm{h}} \mathrm{G}_{\mathrm{h}}$} & \multirow{5}{*}{$\begin{array}{l}x(3)=x(1) \\
y(3)=y(1)\end{array}$} \\
\hline & $y(0)=0$ & $y(1)$ & $y(2)=0.5 y(1)+0.1 y(3)$ & 01 & 54 & & \\
\hline & $x(6)=35$ & $y(7)$ & $x(5)=x(1)+35$ & 67 & 07 & & \\
\hline & $y(6)=0$ & $x(3)$ & $y(5)=y(1)$ & 34 & 64 & & \\
\hline & $y(7)=8.4$ & $y(3)$ & $y(4)=y(2)$ & & & & \\
\hline \multirow{6}{*}{$\mathrm{CG}_{\mathrm{h}} \mathrm{G}_{\mathrm{v}} \mathrm{G}_{\mathrm{h}} \mathrm{CG}_{\mathrm{v}}$} & & & $x(2)=0.15 x(1)+0.5 x(3)$ & & & \multirow{6}{*}{$\mathrm{CG}_{\mathrm{v}} \mathrm{CG}_{\mathrm{v}}$} & \multirow{6}{*}{$\begin{array}{l}x(2)=x(1) \\
y(2)=y(1)\end{array}$} \\
\hline & $x(0)=0$ & $\begin{array}{l}x(1) \\
y(1)\end{array}$ & $y(2)=0.1 y(1)+y(3)$ & 02 & 34 & & \\
\hline & $y(0)=0$ & $\begin{array}{l}y(1) \\
y(2)\end{array}$ & $x(4)=x(3)+0.5 x(1)+15$ & 23 & 86 & & \\
\hline & $x(5)=35$ & $y(2)$ & $y(4)=0.2 y(2)$ & 45 & 07 & & \\
\hline & $y(5)=0$ & $\begin{array}{l}x(2) \\
y(6)\end{array}$ & $x(5)=x(1)+80$ & 13 & 76 & & \\
\hline & & & $y(5)=0.2 y(1)$ & & & & \\
\hline
\end{tabular}

classification of various styles into a graphic data inventory and puts it on the disk. After the designer specifies the interlocking method and the background colour, the system is based on the graphical elements such as line, shape, structure, rhythm, and colour, according to the technical principle of artificial intelligence such as continuous reasoning and campaign judgment strategy. In the graphic combination, a basic unit (pattern) of a graphic is taken out, and according to the data in the basic unit and the interlocking mode and the background colour, the colours of the line group and the interlocking copy parameter are automatically selected within a certain range. Make a copy. After that, the system automatically selects another graphic basic unit to generate a new pattern superimposed on the original screen until the designer artificially terminates the process.
The system can automatically print out all the parameters of the entire graphics generation process.

No matter where the program is running, the system can use the ESC key to interrupt the operation and return to the center point. From the center point you can arbitrarily turn to one of the following ten exits (see Figure 3 ):

(1) End the program run

(2) Select a new interlock method

(3) Modify, select, or specify the following data, parameters, or working methods

(a) Whether to clear the original content of the screen before the interlock copy, and if not clear, the new graphic is superimposed on the original screen 


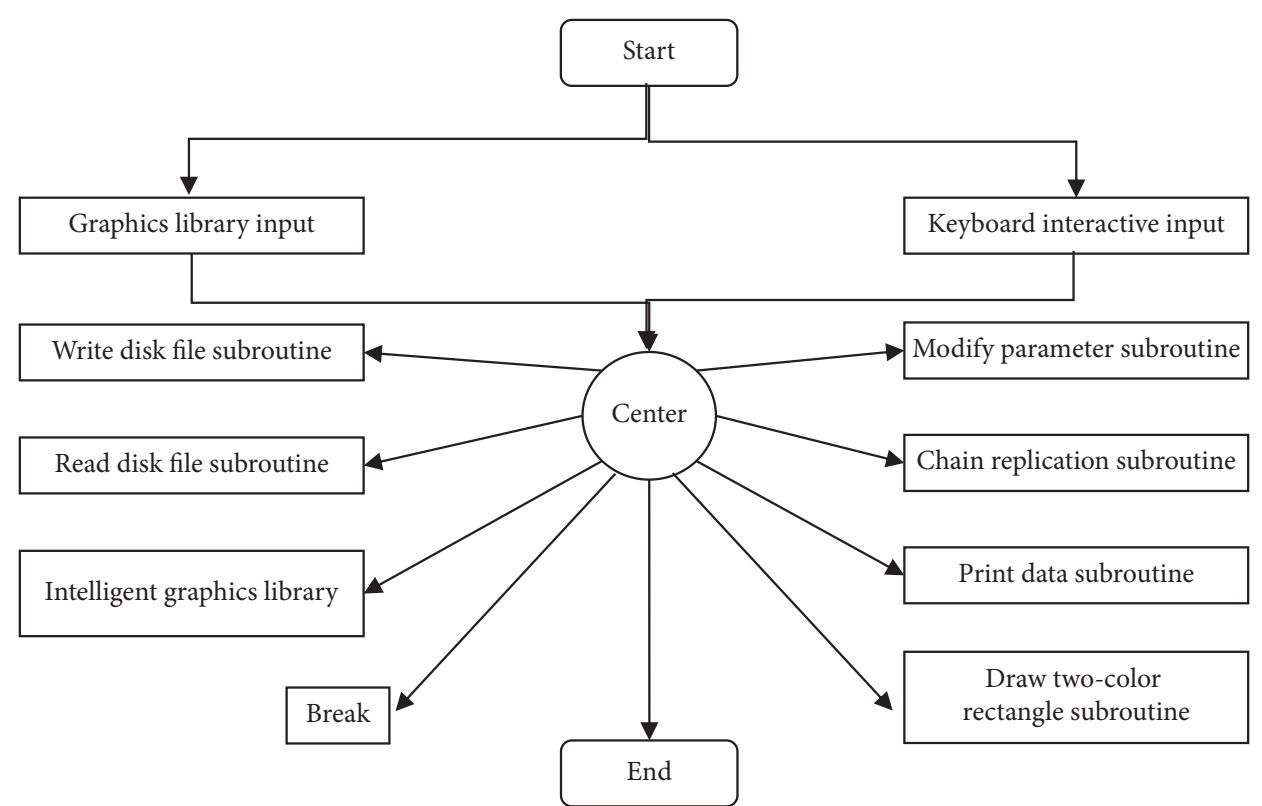

Figure 3: The construction and functions of the system.

(b) Modify the number of interlocking translations $\mathrm{E}, \mathrm{F}$, thereby changing the scaling factor in the $x$ and $y$ directions

(c) Modify the amount of translation of the screen in a certain direction; the initial value is 0

(d) Determine if the graphic is rotated $90^{\circ}$ (i.e., reflecting with a straight line $y=x$ as the axis of symmetry)

(e) Modify the interlock parameters $\mathrm{H}, \mathrm{U}, \mathrm{V}$

(f) Determine whether to add a border on the screen

(g) For high-resolution black and white graphics, you can specify to exchange bright and dark pixels to get a black and white picture

(h) Modify the background colour or background brightness of the low-resolution graphics, or disable the background colour so that the original picture is not destroyed

(i) Modify the colour of any line group

(j) Adjust the colour of all line group

(k) Modify the colour in any enclosed area, and also prohibit colour filling

(4) Print all data of the graphic basic unit and related interlocking copy parameters

(5) Directly transfer to the graphics library working mode, take out new patterns from the graphics library

(6) Use a two-colour rectangular pattern to form a graphic background

(7) Transfer to the AI database to automatically design work methods

(8) All the data of the basic unit of the graphic designed by the interactive method and the necessary interlocking parameters are automatically organized into disk files and stored in the floppy disk according to the specified data structure

(9) Take out the automatically saved graphic file and display it

(10) Suspend the program and transfer to the BASCI standby state to perform the cross-over operation

\section{Result Analysis and Discussion}

This article is from the point of view of the convenience of application, the preparation of a simple program interface testing the algorithm. In program, when we click the menu "Preferences $\longrightarrow$ Draw Graphics [P]", it will pop up "Willow artwork main parameter settings" dialog box. When the parameters are set, clicking on the " $0 \mathrm{~K}$ " button, there will be "parameter confirmation" Prompt message, waiting for choice. When the "Y" button is selected, the drawing graph is displayed. When the "N" button is selected, the parameter needs to be reset. The "Willow Art Main Parameter Setting" dialog box will appear again. If the "Cancel" button is selected, the operating will be given up. When the graph is drawn successfully, if you want to adjust the parameters and obtain the new graph, we can click the menu "Reset $\mathrm{Pa}$ rameters" to reset the parameters. Drawing with current parameters is shown in Figure 4. Willow model is shown in Figure 5.

In order to analyze the script editing method and script stitching algorithm which is better researched in this paper, this dissertation also made an experimental platform for experiment data analysis, which can make accurate statistics and analysis of the data volume and the operation efficiency of the algorithm. For example, Figure 6 shows the experimental analysis platform. 


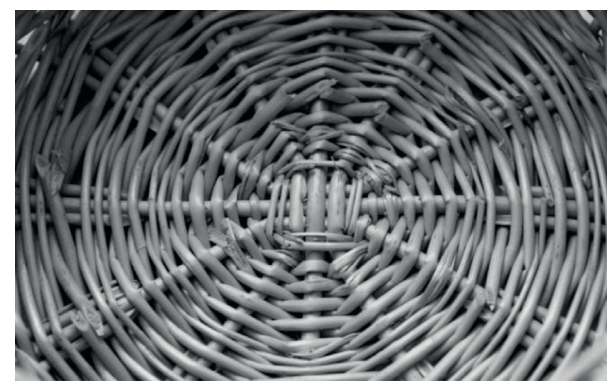

FIGURE 4: Drawing with current parameters.
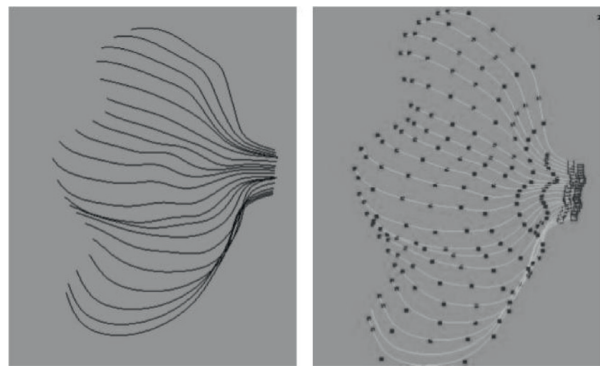

FIGURE 5: Willow model.

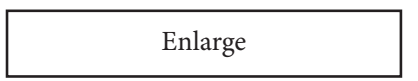

Import XML file 1

Import XML file 2

Draw point

Splice points

Figure 6: The experimental analysis platform.

This platform includes drawing points, zoom in, and calculate run time and other auxiliary functions. In the lab, on the computer of Intel Core $22.66 \mathrm{GHz} \mathrm{CPU}$, 3.SOGB memory, NVIDIA GeForce $8600 \mathrm{GT}$ graphics card, the traditional brute-force algorithm and the research method of this article are tested. Both methods can find the most suitable splicing point accurately. In Figure 7 and Table 3, we can see that the traditional brute force algorithm is not suitable for the scenario studied in this paper. When the amount of calculation data is large, it exceeds 2S. However, the method studied in this paper consumes an exponential increasing trend under the same conditions, but none of them exceeds $2 S$, and the percentage of efficiency improvement is significant, which verifies the effectiveness and efficiency of the proposed algorithm. In order to investigate the effectiveness of our proposal and other methods. Here, we take F1-score into account to assess the experiment results, which can be defined as follows:

$$
\begin{aligned}
f 1-\text { score } & =\frac{2 * \text { precise } * \text { recall }}{\text { precise }+ \text { recall }}, \\
\text { precise } & =\frac{T P}{T P+F P}, \\
\text { recall } & =\frac{T P}{T P+F N} .
\end{aligned}
$$

And we also compared the three methods proposed in the lasted three years, GNNA, RNNA, and CNNA, with our proposal to investigate the effectiveness of our methods. The results can be shown as in Figure 8 . As we can see from Figure 8, the black curve represents the results of our 


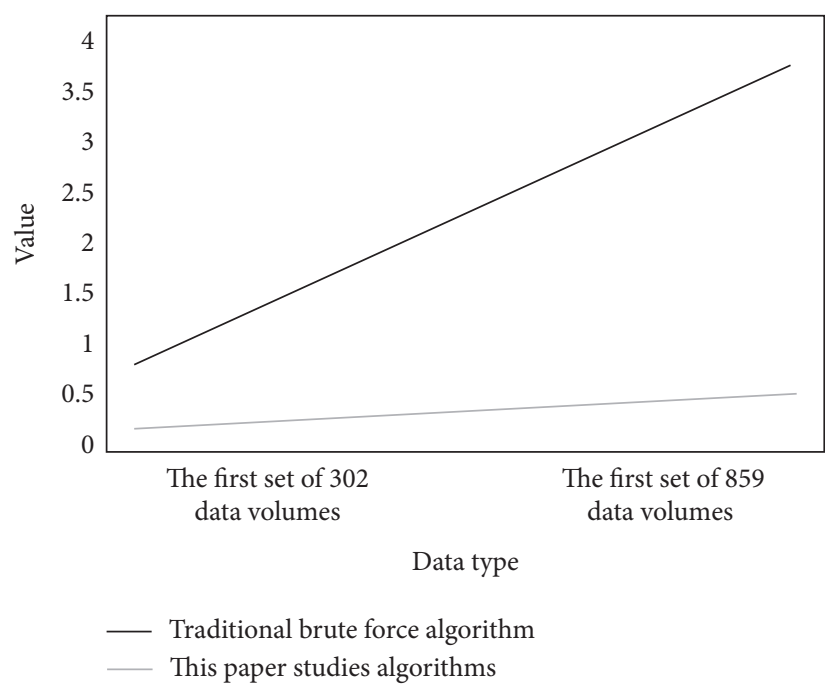

FIgURE 7: The advantages of the algorithm in this paper.

TABLe 3: The advantages of the algorithm.

\begin{tabular}{lccccc}
\hline & $\begin{array}{c}\text { \#node } \\
\text { data }\end{array}$ & $\begin{array}{c}\text { Xml file size } \\
(\mathrm{kB})\end{array}$ & $\begin{array}{c}\text { Traditional brute force algorithm time } \\
\text { consuming }(\mathrm{S})\end{array}$ & $\begin{array}{c}\text { This paper studies the time consuming of } \\
\text { algorithms }(\mathrm{S})\end{array}$ & $\begin{array}{c}\text { Improvement } \\
(\%)\end{array}$ \\
\hline MyXml 1 & 101 & 15 & 0.757 & 0.165 & 76.99 \\
MyXml 2 & 201 & 30 & 1.552 & 0.595 & \multirow{2}{*}{82.85} \\
\hline MyXml 1 & 226 & 24 & 1.721 & 3.470 & \\
MyXml 2 & 633 & 68 & 0.53 & \\
\hline
\end{tabular}

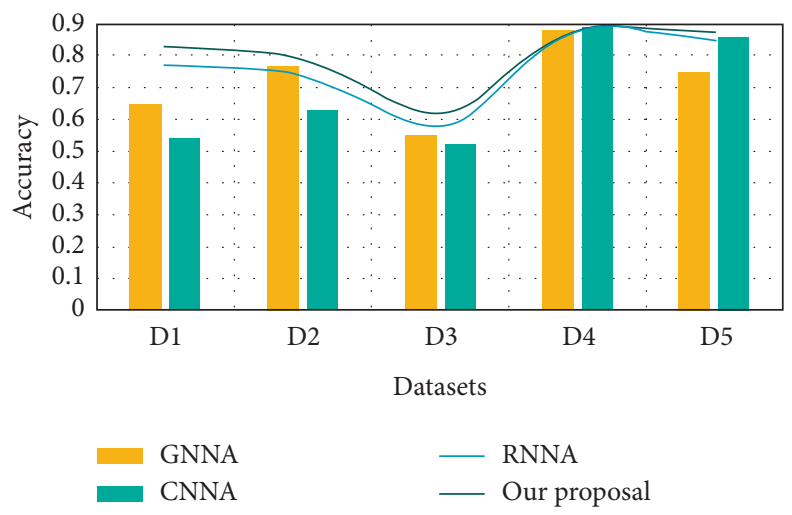

Figure 8: The results of our proposal and other methods.

proposal and others represent the other three methods results. As we can see on all datasets the results of our proposal are better than others except D4, which proves that all methods obtain the same results. GNNA always obtain the worse results which may be caused by the lower generalization, but it can provide more diversities. CNNA, as the 
basic one, can have worst accuracy and lower diversity. RNNA is located at the middle of them. Our proposal can provide high scores and also provide more diversity giving more new products. It indicated that our proposal can perform better than the other three methods.

In summary, the paper introduces the development of postprocessing algorithm of willow technology and starts with the software structure of postprocessing model generation. The realization process of the algorithm is introduced in detail. Then it introduces how to apply the postprocessing platform of animation and splicing the digital shadow script files to form a complete willow craft file. Finally, the effectiveness and efficiency of this algorithm are analyzed, and the usability of the algorithm is further verified and analyzed.

\section{Conclusion}

All in all, the emergence of artificial intelligence has revolutionized the design of computer-aided arts and crafts, not only replacing a lot of tedious and repetitive manual labour, but also improving the quality of production. The continuous improvement of artificial intelligence technology and the generation of various technologies and algorithms make computer-aided arts and crafts design more convenient and efficient, while the efficiency and quality are simultaneously improved, and also pointing out the direction for the development of artificial intelligence technology. The application of artificial intelligence algorithms puts forward great demands on the computing power of computers and our experimental equipment. Therefore, how to further improve the complexity and accuracy of the model is an important topic for our future research.

Intelligence is the main trend of CAPP system development in the future, but from the current level of artificial intelligence technology, it is impossible to make a substantial breakthrough in the intelligent level of CAPP system. To solve the problems of artificial intelligence technology, new breakthroughs must be made in some basic theories and basic sciences, such as in life sciences and mathematics. There must be new breakthroughs in other areas. It can be seen that in the foreseeable future, the development of intelligent CAPP system will still be based on the full use of human intelligence advantages, comprehensive application of various artificial intelligence technologies to achieve intelligent CAPP system. Through the above discussion, we believe that everyone has a certain understanding of the application of computer-aided art design and artificial intelligence and artificial intelligence in computer-aided art design. With the continuous development of artificial intelligence technology, the intelligent CAPP system will further develop the flexibility and effectiveness of knowledge acquisition, expression, and processing; improve the intelligent level of CAPP system; and thus improve the level of modern manufacturing technology. Although our method has achieved good accuracy at present, it is still unable to achieve considerable accuracy in the face of complex environment, and the training time of the model is long. In the future, we will further optimize our model to improve the training speed of the model while ensuring the accuracy.

\section{Data Availability}

The data used to support the findings of this study are included within the article.

\section{Conflicts of Interest}

The authors declare that there are no conflicts of interest regarding the publication of this paper.

\section{Acknowledgments}

This work was supported by the Youth Fund of Humanities and Social Sciences and Art of the Ministry of Education in 2020 (No. 20YJC760014).

\section{References}

[1] R. Rootbernstein, "Arts and crafts as adjuncts to STEM education to foster creativity in gifted and talented students," Asia Pacific Education Review, vol. 16, no. 2, pp. 1-10, 2015.

[2] I. Gatt and S. Karppinen, "An enquiry into primary student teachers' confidence, feelings and attitudes towards teaching arts and crafts in Finland and Malta during initial teacher training," International Journal of Art \& Design Education, vol. 33, no. 1, pp. 75-87, 2014.

[3] T. Petersen, "Computer-aided indexing in the arts: the case for a thesaurus of art terms," Art Libraries Journal, vol. 6, no. 3, pp. 6-11, 2016.

[4] L. Seidenari, G. Serra, A. D. Bagdanov, and A. Del Bimbo, "Local pyramidal descriptors for image recognition," IEEE Transactions on Pattern Analysis and Machine Intelligence, vol. 36, no. 5, pp. 1033-1040, 2014.

[5] P. Vogt, L. Kasper, and J.-P. Burde, "The sound of church bells: tracking down the secret of a traditional arts and crafts trade," The Physics Teacher, vol. 53, no. 7, pp. 438-439, 2015.

[6] Y. Su, X. Chu, D. Chen, and X. Sun, "A genetic algorithm for operation sequencing in CAPP using edge selection based encoding strategy," Journal of Intelligent Manufacturing, vol. 29, no. 9-10, pp. 1-20, 2015.

[7] D. C. Parkes and M. P. Wellman, "Economic reasoning and artificial intelligence," Science, vol. 349, no. 6245, pp. 267-272, 2015.

[8] Z. Ghahramani, "Probabilistic machine learning and artificial intelligence," Nature, vol. 521, no. 7553, pp. 452-459, 2015.

[9] E. S. Rigas, S. D. Ramchurn, and N. Bassiliades, "Managing electric vehicles in the smart grid using artificial intelligence: a survey," IEEE Transactions on Intelligent Transportation Systems, vol. 16, no. 4, pp. 1619-1635, 2015.

[10] M. X. Xia, G. L. Shi, C. Chen et al., "Application and research of reverse engineering and laser manufacturing technology in the arts and crafts," Journal of Guangxi University of Science \& Technology, vol. 8, 2016.

[11] A. Burduk, "Artificial neural networks as tools for controlling production systems and ensuring their stability," Lecture Notes in Computer Science, vol. 8104, pp. 487-498, 2017.

[12] Y. Gao, A. Xu, P. J.-H. Hu, and T.-H. Cheng, "Incorporating association rule networks in feature category-weighted naive Bayes model to support weaning decision making," Decision Support Systems, vol. 96, pp. 27-38, 2017. 
[13] S. Hoochang, H. R. Roth, M. Gao et al., "Deep convolutional neural networks for computer-aided detection: CNN architectures, dataset characteristics and transfer learning," IEEE Transactions on Medical Imaging, vol. 35, no. 5, p. 1285, 2016.

[14] S. Yang, Z. Gong, K. Ye, Y. Wei, Z. Huang, and Z. Huang, "EdgeRNN: a compact speech recognition network with spatio-temporal features for edge computing," IEEE Access, vol. 8, pp. 81468-81478, 2020.

[15] J. Zhang and D. Tao, "Empowering things with intelligence: a survey of the progress, challenges, and opportunities in artificial intelligence of things," IEEE Internet of Things Journal, vol. 8, no. 10, pp. 7789-7817, 2021.

[16] M. S. Hossain and G. Muhammad, "An audio-visual emotion recognition system using deep learning fusion for a cognitive wireless framework," IEEE Wireless Communications, vol. 26, no. 3, pp. 62-68, 2019.

[17] S. P. L. Kumar, J. Jerald, and S. Kumanan, "Feature-based modelling and process parameters selection in a CAPP system for prismatic micro parts," International Journal of Computer Integrated Manufacturing, vol. 28, no. 10, pp. 1046-1062, 2014.

[18] A. B. Yasrebi, A. Hezarkhani, P. Afzal et al., "Application of an ordinary kriging-artificial neural network for elemental distribution in kahang porphyry deposit, Central Iran," Arabian Journal of Geosciences, vol. 13, no. 15, 2020.

[19] R. Ayachi, M. Afif, Y. Said, and M. Atri, "Traffic signs detection for real-world application of an advanced driving assisting system using deep learning," Neural Processing Letters, vol. 51, no. 1, pp. 837-851, 2020.

[20] A. Dey, J. Lahiri, and B. Mukhopadhyaya, "LHC signals of triplet scalars as dark matter portal: cut-based approach and improvement with gradient boosting and neural networks," Journal of High Energy Physics, vol. 2020, no. 6, 2020.

[21] E. Davis and G. Marcus, "Commonsense reasoning and commonsense knowledge in artificial intelligence," Communications of the Acm, vol. 58, no. 9, pp. 92-103, 2015.

[22] M. Stoffel, F. Bamer, and B. Markert, "Neural network based constitutive modeling of nonlinear viscoplastic structural response," Mechanics Research Communications, vol. 95, pp. 85-88, 2019.

[23] F. Khelifi, A. Bradai, A. Benslimane, P. Rawat, and M. Atri, “A survey of localization systems in internet of things," Mobile Networks and Applications, vol. 24, no. 3, pp. 761-785, 2019.

[24] R. J. Duro, J. A. Becerra, J. Monroy, and F. Bellas, "Perceptual generalization and context in a network memory inspired long-term memory for artificial cognition," International Journal of Neural Systems, vol. 29, no. 6, 2019.

[25] B. Soulef, R. Khemiri, F. E. Sayadi, and M. Atri, "Fast CU partition-based machine learning approach for reducing HEVC complexity," Journal of Real-Time Image Processing, vol. 17, 2019.

[26] M. Amini, J. Rezaeenour, and E. Hadavandi, "A neural network ensemble classifier for effective intrusion detection using fuzzy clustering and radial basis function networks," International Journal on Artificial Intelligence Tools, vol. 25, no. 2, 2016.

[27] W. Kristjanpoller and M. C. Minutolo, "Forecasting volatility of oil price using an artificial neural network-GARCH model," Expert Systems with Applications, vol. 65, pp. 233-241, 2016.

[28] V. Vassiliades and C. Christodoulou, "Behavioral plasticity through the modulation of switch neurons," Neural Networks, vol. 74, pp. 35-51, 2016.
[29] B. Javier and J. M. Corchado Rodríguez, "Neural networks in distributed computing and artificial intelligence," Neurocomputing, vol. 272, 2018.

[30] H. C. C. Carneiro, C. E. Pedreira, F. M. G. França, and P. M. V. Lima, "A universal multilingual weightless neural network tagger via quantitative linguistics," Neural Networks, vol. 91, pp. 85-101, 2017.

[31] J. Cussens, M. Järvisalo, J.-H. Korhonen, and M. Bartlett, "Bayesian network structure learning with integer programming: polytopes, facets and complexity," in Proceedings of the Twenty-Sixth International Joint Conference on Artificial Intelligence, Melbourne, Australia, August 2017. 